\title{
STATISTICAL MEASUREMENT OF THE GAMMA-RAY SOURCE-COUNT DISTRIBUTION AS A FUNCTION OF ENERGY
}

\author{
Hannes-S. Zechlin ${ }^{1,2}$, Alessandro Cuoco ${ }^{2,3}$, Fiorenza Donato ${ }^{1,2}$, Nicolao Fornengo ${ }^{1,2}$, And Marco Regis ${ }^{1,2}$ \\ ${ }^{1}$ Dipartimento di Fisica, Università di Torino, via P. Giuria, 1, I-10125 Torino, Italy; zechlin@to.infn.it \\ 2 Istituto Nazionale di Fisica Nucleare, Sezione di Torino, via P. Giuria, 1, I-10125 Torino, Italy \\ ${ }^{3}$ Institute for Theoretical Particle Physics and Cosmology (TTK), RWTH Aachen University, D-52056 Aachen, Germany \\ Received 2016 May 13; revised 2016 June 23; accepted 2016 July 1; published 2016 July 29
}

\begin{abstract}
Statistical properties of photon count maps have recently been proven as a new tool to study the composition of the gamma-ray sky with high precision. We employ the 1-point probability distribution function of six years of FermiLAT data to measure the source-count distribution $d N / d S$ and the diffuse components of the high-latitude gammaray sky as a function of energy. To that aim, we analyze the gamma-ray emission in five adjacent energy bands between 1 and $171 \mathrm{GeV}$. It is demonstrated that the source-count distribution as a function of flux is compatible with a broken power law up to energies of $\sim 50 \mathrm{GeV}$. The index below the break is between 1.95 and 2.0 . For higher energies, a simple power-law fits the data, with an index of $2.2_{-0.3}^{+0.7}$ in the energy band between 50 and $171 \mathrm{GeV}$. Upper limits on further possible breaks as well as the angular power of unresolved sources are derived. We find that point-source populations probed by this method can explain $83_{-13}^{+7} \%\left(81_{-19}^{+52} \%\right)$ of the extragalactic gamma-ray background between 1.04 and $1.99 \mathrm{GeV}$ (50 and $171 \mathrm{GeV}$ ). The method has excellent capabilities for constraining the gamma-ray luminosity function and the spectra of unresolved blazars.
\end{abstract}

Key words: gamma rays: diffuse background - gamma rays: general - methods: statistical

Supporting material: data behind figure

\section{INTRODUCTION}

The study of gamma-ray sources is one of the main tasks in understanding the gamma-ray sky. Compact emission regions and high distances cause most extragalactic sources to appear point-like in current measurements (Acero et al. 2015), in distinction to truly diffuse components such as Galactic foreground (GF) emission (Acero et al. 2016) or unresolved components like the isotropic diffuse gamma-ray background (IGRB; Ackermann et al. 2015). Source populations are characterized by individual source-count distributions, which encode their physical and evolutional properties as functions of the integral flux $S$. Since different source classes, e.g., flatspectrum radio quasars (FSRQs), BL Lacertae (BL Lac) objects, misaligned active galactic nuclei (mAGNs), and starforming galaxies (SFGs) distinguish themselves by spectral index, distance, and luminosity (e.g., Massaro et al. 2016), the combined gamma-ray source-count distribution $d N / d S$ is nontrivial and depends on the energy band considered.

For the energy band between 1 and $10 \mathrm{GeV}$, Zechlin et al. (2016, henceforth Z16) have recently demonstrated that statistical methods can be used to measure the combined $d N / d S$ with high accuracy and with sensitivity down to fluxes about one order of magnitude below catalog detection thresholds. The six-year data provided by the Fermi Large Area Telescope (Fermi-LAT; Atwood et al. 2009; Ackermann et al. 2012a) have been used to determine $d N / d S$ and the composition of the gamma-ray sky at high Galactic latitudes $\left(|b| \geqslant 30^{\circ}\right)$. The analysis was based on a global fit of the 1-point probability distribution function (1pPDF) of the LAT photon counts map. The method of analyzing the simple 1pPDF (cf. Malyshev \& Hogg 2011) has been extended to include spatial templates, providing significantly higher sensitivity. Furthermore, the 1pPDF method does not introduce biases in the measured $d N / d S$ distribution near the catalog detection threshold. A similar analysis has been recently applied to the Galactic Center region (Lee et al. 2016).

In this Letter, we extend the analysis of Z16 to five energy bands, covering the range from 1.04 to $171 \mathrm{GeV}$, thus measuring $d N / d S$ as a function of energy. We use the same data and methods described in Z16. The measurements are compared to the $d N / d S$ distribution predicted by fiducial models of extragalactic source populations and implications are discussed.

\section{METHOD AND DATA}

The gamma-ray sky was modeled with a superposition of three components: (i) an isotropic distribution of point sources $d N / d S$, (ii) a contribution from GF emission, and (iii) a truly isotropic component, representing sources too faint to be seen by the $1 \mathrm{pPDF}$ method or residual cosmic-ray contamination. For each energy band, the $d N / d S$ distribution was approximated with a multiply broken power law (MBPL) with $N_{\mathrm{b}}$ breaks $S_{\mathrm{b} j}, j=1,2, \ldots, N_{\mathrm{b}}$,

$$
\frac{d N}{d S} \propto\left\{\begin{array}{lc}
\left(\frac{S}{S_{0}}\right)^{-n_{1}}, & S>S_{\mathrm{b} 1} \\
\left(\frac{S_{\mathrm{b} 1}}{S_{0}}\right)^{-n_{1}+n_{2}}\left(\frac{S}{S_{0}}\right)^{-n_{2}}, & S_{\mathrm{b} 2}<S \leqslant S_{\mathrm{b} 1} \\
\vdots & \vdots \\
\left(\frac{S_{\mathrm{b} 1}}{S_{0}}\right)^{-n_{1}+n_{2}}\left(\frac{S_{\mathrm{b} 2}}{S_{0}}\right)^{-n_{2}+n_{3}} \cdots\left(\frac{S}{S_{0}}\right)^{-n_{N_{\mathrm{b}}+1}}, \\
& S \leqslant S_{\mathrm{b} N_{\mathrm{b}},}
\end{array}\right.
$$

where $S_{0}$ is a constant reference flux and the indices of the power-law components are denoted by $n_{j}$. For simplicity, the notation omits a separate index denoting the energy dependence of $d N / d S$. The $1 \mathrm{pPDF}$ was computed following the method of Z16. Exposure inhomogeneities were accounted for 
Table 1

Energy Bands and Analysis Parameters

\begin{tabular}{lccccccc}
\hline \hline $\begin{array}{l}E_{\min } \\
(\mathrm{GeV})\end{array}$ & $\begin{array}{c}E_{\max } \\
(\mathrm{GeV})\end{array}$ & $\begin{array}{c}|b| \\
\left({ }^{\circ}\right)\end{array}$ & $\kappa$ & $\begin{array}{c}\sigma_{\mathrm{psf}} \\
\left.{ }^{\circ}\right)\end{array}$ & $\Gamma$ & $\begin{array}{c}S_{0} / 10^{-9} \\
\left(\mathrm{~cm}^{-2} \mathrm{~s}^{-1}\right)\end{array}$ & $N_{\mathrm{b}}^{\mathrm{h}}$ \\
\hline 1.04 & 1.99 & $\geqslant 30$ & 6 & 0.52 & 2.4 & 30 & $1,2,3$ \\
1.99 & 5.0 & $\geqslant 30$ & 6 & 0.31 & 2.4 & 5 & $1,2,3$ \\
5.0 & 10.4 & $\geqslant 30$ & 6 & 0.23 & 2.4 & 1 & 1,2 \\
10.4 & 50.0 & $\geqslant 30$ & 6 & 0.15 & 2.2 & 0.1 & 1,2 \\
50 & 171 & $\geqslant 10$ & 7 & 0.13 & 2.2 & 0.1 & 1,2 \\
\hline
\end{tabular}

Note. For each energy band $\left[E_{\min }, E_{\max }\right]$, the table lists the considered Galactic latitude cut $|b|$, the resolution of the HEALPix pixelization $\kappa$, where the number of pixels of an all-sky map is given by $12 \times 2^{2 \kappa}$, and the $68 \%$ containment radius of the effective PSF, $\sigma_{\mathrm{psf}}$. Point sources were assumed to emit powerlaw-type energy spectra with photon index $\Gamma$. The reference flux chosen for the MBPL parameterization is given by $S_{0}$. The last column lists the numbers of free breaks considered for the hybrid approach.

by slicing the map into $N_{\exp }=20$ regions separated by isocontours of equal exposure. The GF and the modeling of the diffuse isotropic background component were treated in the same way as in Z16. ${ }^{4}$ Corrections of $d N / d S$ for the finite pointspread function (PSF) of the instrument were computed consistently for each energy band considered in this analysis. The data were fit as described in Z16, adopting the definition of the likelihood function $\mathcal{L}_{2}(\Theta)$ (see Z16, Equation (17)). Besides the normalization of $d N / d S$, the vector of free parameters $\Theta$ contains the breaks $S_{\mathrm{bj} j}$, the indices $n_{j}$, an overall normalization $A_{\text {gal }}$ of the GF template, and the (integral) flux $F_{\text {iso }}$ of the diffuse isotropic background component. The likelihood was sampled using MultiNest (Feroz et al. 2009). We used the frequentist parameter estimation approach, based on the profile likelihood function as derived from the likelihood samples obtained.

We considered Fermi-LAT data (P7REP_CLEAN) covering the first six years of the mission science operations for five adjacent energy bands. Table 1 lists the energy bands and corresponding analysis parameters.

The choice of the energy bands was motivated by the analysis of gamma-ray anisotropies in the high-latitude sky; see Ackermann et al. (2012c). The highest-energy band matches the first energy bin quoted in the 2FHL catalog (Ackermann et al. 2016b). Event selection and data processing were carried out as outlined in Z16 for every individual energy band. We allowed a maximum zenith angle of $90^{\circ}$ and the rocking angle of the satellite was constrained to values smaller than $52^{\circ}$. To avoid unnecessary broadening of the effective PSF, we restricted the event selection to FRONT-converting events for the two lowest-energy bands, while for higher energies all events were used. The resolution parameter $\kappa$ of the HEALPix pixelization (Górski et al. 2005) was chosen to undersample the effective PSF (see Section 3 in Z16). We compared the resolutions $\kappa=6$ and $\kappa=7$, adopting the one providing higher sensitivity. Source spectral energy distributions were assumed to follow power laws. The average spectral photon index $\Gamma$ was selected following Abdo et al. (2010). ${ }^{5}$ For all but

\footnotetext{
4 Masking Galactic structures such as the Fermi Bubbles or Galactic Loop I did not significantly affect the results.

5 We checked that systematic uncertainties related to this choice are small, by varying $\Gamma$ between 2.1 and 2.4 .
}

the last energy band we restricted the analysis to Galactic latitudes $|b| \geqslant 30^{\circ}$. Due to significantly fewer events, we chose a Galactic latitude cut of $|b| \geqslant 10^{\circ}$ for the highest-energy band. Indeed, in this band, the GF is less prominent, its spectrum being softer than the source component.

\subsection{Source-count Distribution Fit}

To fit our model of the gamma sky to the data, we used the analysis chain developed by Z16, i.e., $d N / d S$ was parameterized with a pure MBPL (MBPL approach) and an improved representation incorporating an additional node (hybrid approach). A node is defined as a break at a fixed position, chosen at the faint end of $d N / d S$, with the normalization left free to vary. The reference flux $S_{0}$ of the MBPL representation of $d N / d S$ was chosen for each energy band separately (see Table 1). Stability was checked by varying $S_{0}$ within a factor of 10. We compared MBPL parameterizations of the $d N / d S$ distribution with up to three free breaks, depending on the energy band.

\section{RESULTS AND DISCUSSION}

\subsection{Source-count Distribution}

The MBPL approach was employed uniformly for each energy band, comparing $d N / d S$ parameterizations with two and three free breaks. The data were described sufficiently by twobreak fits for all energy bands, i.e., no statistical preference for three breaks was found, with the test statistic TS $=-2 \Delta \ln \mathcal{L}_{2}$ between the two hypotheses at most reaching a value of 2 . As discussed in Z16, below the second (third) break the fit generally prefers a sharp cutoff, which we interpret as a loss of sensitivity of the method (as opposed to an intrinsic feature of the $d N / d S$ distribution).

To improve the estimation of uncertainty bands, we employed the hybrid approach by adding a node $S_{\text {nd }}$, imposing the faint cutoff positions found with the MBPL approach. We chose nodes at $3 \times 10^{-12}, 10^{-12}, 5 \times 10^{-13}, 3 \times 10^{-13}$, and $5 \times 10^{-14} \mathrm{~cm}^{-2} \mathrm{~s}^{-1}$ for the bands as ordered by increasing energy. The power-law index below the node was fixed to -10 . Given the high number of events detected in the first two energy bands ${ }^{6}$, we considered $d N / d S$ parameterizations with one, two, and three free breaks (and a node), see Table 1, as driven by results of Z16. Due to significantly fewer events at higher energies and the resulting sensitivity decrease, only one or two free breaks were used for the last three energy bands.

The results are shown in Figure 1. Marginalized parameter estimates are listed in Table 2 . In addition, the best-fit $d N / d S$ distributions and the corresponding uncertainty bands are provided as supporting material.

As for the MBPL case, we found that the additional breaks did not significantly improve the fit for any of the five energy bands. The data were described sufficiently well by $d N / d S$ distributions with a single break at comparably high fluxes and a node at the faint end. The best fits for this case are depicted in Figure 1 by the solid blue lines, which are shown only above the estimated sensitivity of the analysis (see below). On the contrary, to have a more robust and realistic estimate of the uncertainty bands we keep the band resulting from the fits with multiple breaks (for the three energy bands below $10 \mathrm{GeV}$ ).

\footnotetext{
6 Total number of selected events, ordered by energy: 487,854, 268,261, $119,123,57,571$, and 10,005 .
} 


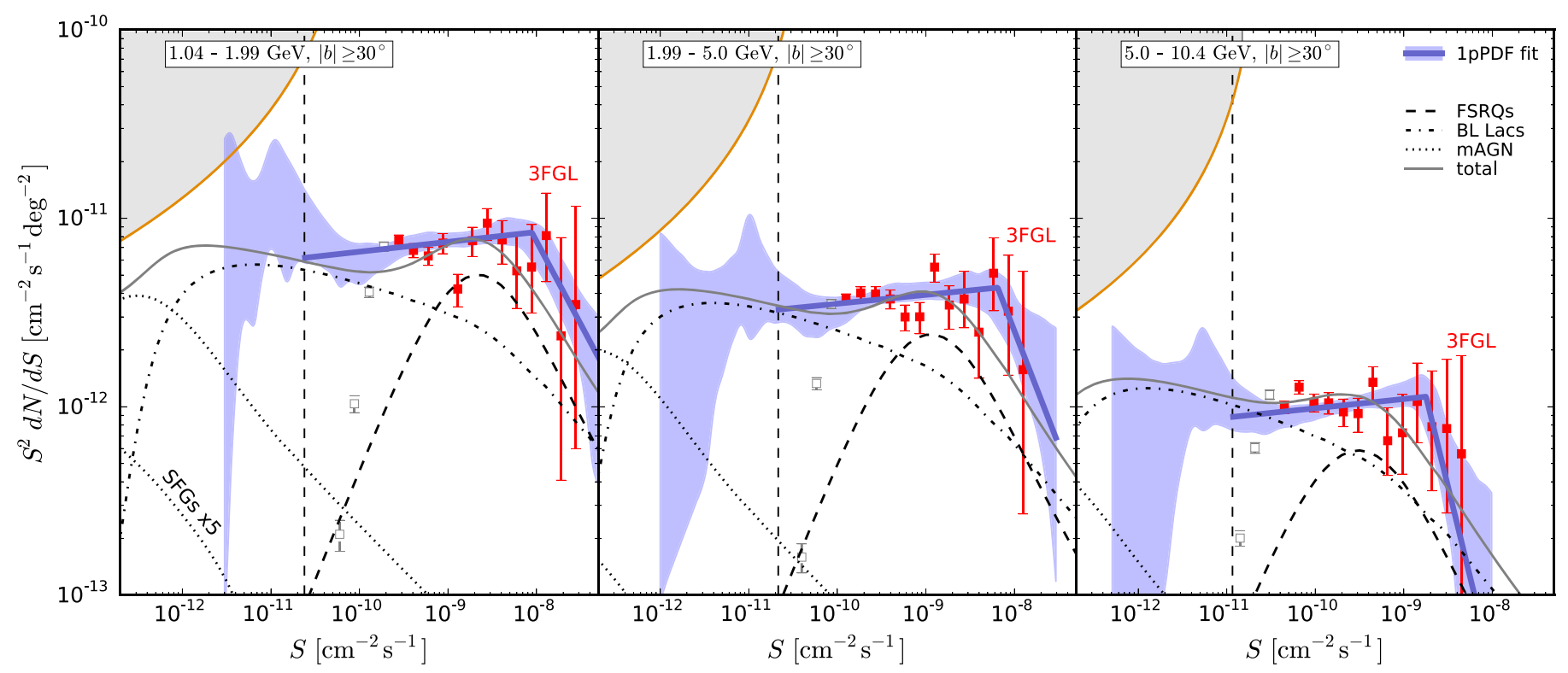

(a)

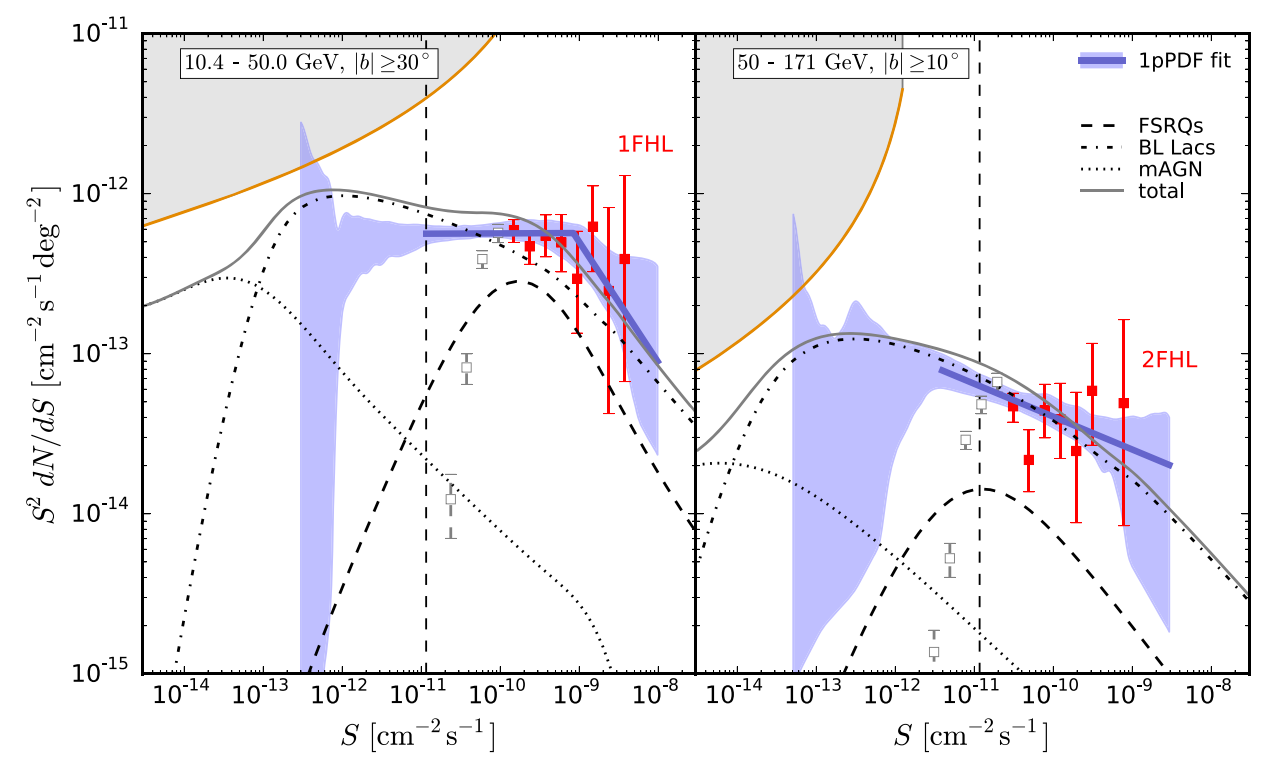

(b)

Figure 1. Differential source-count distributions $d N / d S$ obtained from six-year Fermi-LAT data with the 1pPDF method. The data have been fit in five energy bands using the hybrid approach. The best fits and the uncertainties at $68 \%$ confidence level are depicted by the solid blue lines and the blue shaded bands. The fits are compared to the $d N / d S$ distributions derived from cataloged sources (red squares; Poissonian errors following Gehrels 1986). The open gray squares depict $d N / d S$ points from sources below the nominal detection threshold, where the sample suffers from catalog incompleteness. Thus, they cannot be used for comparison. The vertical dashed lines indicate the sensitivity estimates $S_{\text {sens }}$. The orange lines, bordering the gray shaded areas, mark the region where the contribution from point sources equals $F_{\mathrm{ps}}=F_{\mathrm{tot}}-F_{\mathrm{gal}}$. In this case, $F_{\mathrm{ps}}$ has been derived with Equation (3), but extrapolating the best-fit $d N / d S$ distributions with a power-law component of varying index below the position of the last free break. The orange lines therefore constrain the position of a next break, given the condition $F_{\mathrm{ps}} \leqslant F_{\text {tot }}-F_{\text {gal }}$. The dashed, dot-dashed, and dotted lines depict model predictions for FSRQs (Ajello et al. 2012), BL Lacs (Ajello et al. 2014; model LDDE 1 ), and mAGNs (Di Mauro et al. 2014a), respectively. The model of SFGs has been taken from Ackermann et al. (2012b). The solid gray lines denote the sum of these contributions.

(The data used to create this figure are available).

These bands are plotted as blue shaded regions in the figure. The resulting $d N / d S$ distributions are compared to counts of cataloged point sources $^{7}$, derived from the 3FGL (Acero

\footnotetext{
7 The method of deriving $d N / d S$ for cataloged sources is explained in Z16, Section 4.3.5.
}

et al. 2015), 1FHL (Ackermann et al. 2013), and 2FHL (Ackermann et al. 2016b) source catalogs, respectively.

As demonstrated in the figure, the 1pPDF fits match the $d N / d S$ distributions of cataloged sources well within uncertainties. The $1 \mathrm{pPDF}$ method allows us to measure the energydependent $d N / d S$ in the regime of undetected faint point sources down to integral fluxes of $\sim 10^{-11} \mathrm{~cm}^{-2} \mathrm{~s}^{-1}$, which are 
Table 2

$d N / d S$ Parameters and Sky Composition

\begin{tabular}{|c|c|c|c|c|c|}
\hline Parameter $^{\mathrm{a}}$ & $1.04-1.99 \mathrm{GeV}$ & $1.99-5.0 \mathrm{GeV}$ & $5.0-10.4 \mathrm{GeV}$ & $10.4-50.0 \mathrm{GeV}$ & $50-171 \mathrm{GeV}$ \\
\hline $\begin{array}{l}S_{\mathrm{b} 1} \\
n_{1} \\
n_{2}\end{array}$ & $\begin{array}{c}9_{-5}^{+10} \times 10^{-9} \\
2.88_{-0.47}^{+0.83} \\
1.95_{-0.07}^{+0.06}\end{array}$ & $\begin{array}{c}7_{-5}^{+13} \times 10^{-9} \\
3.21_{-1.16}^{+1.09} \\
1.95_{-0.06}^{+0.07}\end{array}$ & $\begin{array}{c}2_{-1}^{+2} \times 10^{-9} \\
3.92_{-1.53}^{+1.72} \\
1.95_{-0.09}^{+0.13}\end{array}$ & $\begin{array}{c}8_{-8}^{+21} \times 10^{-10} \\
2.75_{-0.68}^{+0.21} \\
2.00_{-0.28}^{+0.21}\end{array}$ & $\begin{array}{c}\cdots \\
2.21_{-0.29}^{+0.69} \\
\ldots\end{array}$ \\
\hline $\begin{array}{l}F_{\mathrm{ps}} \\
F_{\text {gal }} \\
F_{\text {iso }} \\
F_{\text {tot }} \\
q_{\mathrm{ps}} \\
q_{\text {gal }} \\
q_{\text {iso }}\end{array}$ & $\begin{array}{c}2.5_{-0.4}^{+0.2} \times 10^{-7} \\
6.54_{-0.05}^{+0.02} \times 10^{-7} \\
1.8_{-0.8}^{+3.9} \times 10^{-8} \\
9.17(1) \times 10^{-7} \\
0.27_{-0.04}^{+0.02} \\
0.714_{-0.005}^{+0.003} \\
0.02_{-0.01}^{+0.04}\end{array}$ & $\begin{array}{c}1.24_{-0.25}^{+0.07} \times 10^{-7} \\
3.24_{-0.03}^{+0.02} \times 10^{-7} \\
5.6_{-0.6}^{+27.8} \times 10^{-9} \\
4.573(9) \times 10^{-7} \\
0.27_{-0.06}^{+0.02} \\
0.708_{-0.006}^{+0.005} \\
0.012_{-0.001}^{+0.061}\end{array}$ & $\begin{array}{c}2.7_{-0.3}^{+0.8} \times 10^{-8} \\
6.59_{-0.10}^{+0.07} \times 10^{-8} \\
1.8_{-0.7}^{+0.3} \times 10^{-8} \\
1.103(3) \times 10^{-7} \\
0.24_{-0.03}^{+0.08} \\
0.598_{-0.01}^{+0.007} \\
0.16_{-0.07}^{+0.03}\end{array}$ & $\begin{array}{c}1.4_{-0.1}^{+0.6} \times 10^{-8} \\
2.60_{-0.07}^{+0.04} \times 10^{-8} \\
1.2_{-0.8}^{+0.2} \times 10^{-8} \\
5.27(2) \times 10^{-8} \\
0.27_{-0.03}^{+0.11} \\
0.494_{-0.0013}^{+0.008} \\
0.23_{-0.15}^{+0.04}\end{array}$ & $\begin{array}{c}1.7_{-0.4}^{+1.1} \times 10^{-9} \\
2.75_{-0.10}^{+0.07} \times 10^{-9} \\
1.2_{-0.9}^{+0.5} \times 10^{-9} \\
5.67(6) \times 10^{-9} \\
0.29_{-0.08}^{+0.19} \\
0.49_{-0.02}^{+0.01} \\
0.22_{-0.17}^{+0.10}\end{array}$ \\
\hline $\begin{array}{l}S_{\mathrm{b} 1}^{\mathrm{UL}}\left(\Delta n_{12}>0.3\right) \\
S_{\mathrm{b} 2}^{\mathrm{UL}}\left(\Delta n_{23}>0.3\right) \\
C_{\mathrm{P}}\left(S_{\mathrm{th}}^{3 \mathrm{FGL}}\right) \\
C_{\mathrm{P}}\left(S_{\mathrm{th}}^{\mathrm{fix}}\right)\end{array}$ & $\begin{array}{c}\cdots \\
2.3 \times 10^{-10} \\
2.3_{-0.1}^{+0.7} \times 10^{-18} \\
4.2_{-0.1}^{+0.7} \times 10^{-18}\end{array}$ & $\begin{array}{c}\cdots \\
1.7 \times 10^{-10} \\
6.1_{-0.4}^{+2.2} \times 10^{-19} \\
1.06_{-0.04}^{+0.22} \times 10^{-18}\end{array}$ & $\begin{array}{c}\cdots \\
1.5 \times 10^{-10} \\
5_{-1}^{+1} \times 10^{-20} \\
1.3_{-0.1}^{+0.1} \times 10^{-19}\end{array}$ & $\begin{array}{c}\cdots \\
3.3 \times 10^{-11} \\
2.3_{-0.5}^{+0.4} \times 10^{-20} \\
1.8_{-0.1}^{+0.1} \times 10^{-19}\end{array}$ & $\begin{array}{c}1.3 \times 10^{-11} \\
\ldots \\
2_{-4}^{+3} \times 10^{-22} \\
4.4_{-0.7}^{+0.3} \times 10^{-21}\end{array}$ \\
\hline
\end{tabular}

Note. Selection of parameter values obtained for different energy bands. The parameters of the $d N / d S$ distribution correspond to a parameterization with one break $S_{\mathrm{b} 1}$ and a node. Parentheses denote symmetric errors on the preceding digit. The quantities $q_{\mathrm{ps}}, q_{\mathrm{gal}}$, and $q_{\text {iso }}$ are the ratios of the integral flux components and $F_{\text {tot }}$. The upper limits on a first $\left(S_{\mathrm{b} 1}^{\mathrm{UL}}\right)$ or second $\left(S_{\mathrm{b} 2}^{\mathrm{UL}}\right)$ intrinsic break are at the $95 \%$ confidence level. The anisotropy $C_{\mathrm{P}}$ is given for two different point-source detection thresholds: $C_{\mathrm{P}}\left(S_{\mathrm{th}}^{3 \mathrm{FGL}}\right)$ denotes the anisotropy for the effective detection threshold $S_{\mathrm{th}}^{3 \mathrm{FGL}}$ of the $3 \mathrm{FGL}$ catalog. Since it is difficult to explicitly define $S_{\mathrm{th}}^{3 \mathrm{FGL}}$, the corresponding anisotropy has been estimated as $C_{\mathrm{P}}\left(S_{\mathrm{th}}^{3 \mathrm{FGL}}\right) \approx C_{\mathrm{P}}\left(S_{\mathrm{th}}^{<1}\right)-C_{\mathrm{P}}^{\text {cat }}\left(S_{\mathrm{th}}^{<1}\right)$, where $C_{\mathrm{P}}$ refers to Equation $(2), C_{\mathrm{P}}^{\text {cat }}$ denotes the anisotropy contributed by cataloged (i.e., resolved) sources only, and $S_{\mathrm{th}}^{<1}$ approximates the flux below which the detection efficiency of the 3FGL catalog becomes much less than 1 . For $S_{\mathrm{th}}^{<1}$, the values $2 \times 10^{-10}, 10^{-10}, 4 \times 10^{-11}, 2 \times 10^{-11}$, and $8 \times 10^{-12} \mathrm{~cm}^{-2} \mathrm{~s}^{-1}$ (from left to right) have been used. The results are stable against choosing higher values of $S_{\mathrm{th}}^{<1}$. On the contrary, $S_{\mathrm{th}}^{\text {fix }}$ denotes a sharp threshold, approximating the flux above which the catalogs used in Figure 1 have full detection efficiency, i.e., $2 \times 10^{-10}, 10^{-10}, 4 \times 10^{-11}, 10^{-10}$, and $2 \times 10^{-11} \mathrm{~cm}^{-2} \mathrm{~s}^{-1}$.

${ }^{\mathrm{a}}$ The break $S_{\mathrm{b} 1}$ and the upper limits $S_{\mathrm{b} j}^{\mathrm{UL}}$ are given in units of $\mathrm{cm}^{-2} \mathrm{~s}^{-1}$. The integral fluxes $F_{\mathrm{ps}}, F_{\mathrm{gal}}, F_{\mathrm{iso}}$, and $F_{\mathrm{tot}}$ are in units of $\mathrm{cm}^{-2} \mathrm{~s}^{-1} \mathrm{sr}^{-1}$. The unit of the anisotropy $C_{\mathrm{P}}$ is $\left(\mathrm{cm}^{-2} \mathrm{~s}^{-1} \mathrm{sr}^{-1}\right)^{2} \mathrm{sr}$.

typically an order of magnitude below the nominal catalog detection threshold, below which the catalog detection efficiency is much less than 1 . The uncertainty bands of the fits significantly broaden below the sensitivity limit of $\sim 10^{-11} \mathrm{~cm}^{-2} \mathrm{~s}^{-1}$. The sensitivity can be compared to an analytic estimate $S_{\text {sens }}$, corresponding to two photons per pixel, indicated by the dashed vertical lines in Figure 1 (see Z16, Section 4). The actual sensitivity matches these expectations. In the 50 to $171 \mathrm{GeV}$ band, the actual sensitivity is better by a factor of $2-3$.

We conclude that the $d N / d S$ distributions in the four bands below $50 \mathrm{GeV}$ are compatible with broken power laws for fluxes above $\sim 10^{-11} \mathrm{~cm}^{-2} \mathrm{~s}^{-1}$. The power-law index $n_{2}$ below the break is compatible with values between 1.95 and 2.0. The $d N / d S$ distribution in the highest-energy band between 50 and $171 \mathrm{GeV}$ is compatible with a simple power law ${ }^{8}$ with an index $2.2_{-0.3}^{+0.7}$. Within uncertainties, this index is compatible with the 2FHL catalog $d N / d S$ of Ackermann et al. (2016a), who conducted a catalog analysis of Pass 8 data between $50 \mathrm{GeV}$ and $2 \mathrm{TeV}$.

Given the absence of a significant second (first, for the last band) intrinsic break of $d N / d S$, we derived corresponding upper limits (cf. Z16). In this case, we assumed that a break would be present if the indices of the power-law components above and below the break differed by $\Delta n_{i+1}=\left|n_{i}-n_{i+1}\right|>0.3$, for $i=2(1)$. The upper limits at the $95 \%$ confidence level are quoted in Table 2. All upper limits are either located at or below the detection thresholds of current catalogs. For the highest-energy band between 50 and

8 That is, the fit did not prefer a break significantly above the sensitivity limit.
$171 \mathrm{GeV}$, any break has been constrained to be at fluxes below $1.3 \times 10^{-11} \mathrm{~cm}^{-2} \mathrm{~s}^{-1}$. This upper limit is consistent with the break found in Ackermann et al. (2016a).

\subsection{Anisotropies}

The anisotropy (or autocorrelation angular power spectrum) provides a complementary measure of unresolved point sources (Ackermann et al. 2012c; Cuoco et al. 2012; Di Mauro et al. 2014b; Ripken et al. 2014). For a given $d N / d S$, the anisotropy can be calculated (Cuoco et al. 2012) as

$$
C_{\mathrm{P}}\left(S_{\mathrm{th}}\right)=\int_{0}^{S_{\mathrm{th}}} S^{2} \frac{d N}{d S} d S,
$$

where $S_{\text {th }}$ is the flux threshold of individually resolved (detected) point sources. In our case, the integral is effectively limited to the interval $\left[S_{\mathrm{nd}}, S_{\mathrm{th}}\right]$, given that the $d N / d S$ distribution was parameterized with a sharp cutoff below the node $S_{\text {nd. }}$. Table 2 lists the resulting anisotropies corresponding to our $d N / d S$ fits, assuming flux thresholds approximating the catalog detection thresholds (quoted in the caption of the table), with the aim of comparing them with new anisotropy measurements available in the near future (M. Fornasa et al. 2016 , in preparation).

\subsection{Composition of the Gamma-Ray Sky}

The contribution of point sources to the total flux $F_{\text {tot }}$ of the region of interest (ROI) in the energy band $\left[E_{\min }, E_{\max }\right]$ is given 
by

$$
F_{\mathrm{ps}}=\int_{0}^{S_{\mathrm{cut}}} S \frac{d N}{d S} d S
$$

which again is effectively limited to a lower bound of $S_{\text {nd }}$ in our case. The full posterior was employed to derive the profile likelihood of $F_{\mathrm{ps}}$. The GF contribution $F_{\mathrm{gal}}$ was obtained from the integral template flux and the normalization parameter $A_{\text {gal }}$. The diffuse isotropic background component $F_{\text {iso }}$ was already one of the fit parameters. The sum of the three components can be compared to $F_{\text {tot, }}$, which was independently derived from integrating the events map divided by the energy-averaged exposure map over the ROI.

Table 2 lists the composition of the high-latitude gamma-ray sky for each energy band. The contribution of point sources $F_{\mathrm{ps}}$ can be compared to the extragalactic gamma-ray background (EGB), $F_{\mathrm{EGB}}$, as measured in Ackermann et al. (2015). The resulting fractional contributions $F_{\mathrm{ps}} / F_{\mathrm{EGB}}$ in each energy band are $0.83_{-0.13}^{+0.07}, 0.79_{-0.16}^{+0.04}, 0.66_{-0.07}^{+0.20}, 0.66_{-0.05}^{+0.28}$, and $0.81_{-0.19}^{+0.52}$, respectively.

\subsection{Comparison with Models}

In order to assess the power of this method, we compare our $d N / d S$ measurement as a function of energy with state-of-theart models. We consider all source classes known to provide major contributions to the EGB. The blazar gamma-ray luminosity function (GLF) and spectrum are modeled following Ajello et al. (2012) for FSRQs, Ajello et al. (2014) and Di Mauro et al. (2014c) for BL Lacs, and Ajello et al. (2015) when considering a single description for all blazars. Misaligned AGNs are taken from Di Mauro et al. (2014a), while for SFGs we followed Ackermann et al. (2012b) with an infrared luminosity function from Gruppioni et al. (2013). The absorption due to extragalactic background light is modeled according to Finke et al. (2010), affecting the two highestenergy bands.

The inset of Figure 2 shows that all the different descriptions adopted for blazars are compatible with the cataloged integral source-count distribution. However, they can have significantly different behaviors for unresolved sources.

The sum of the model predictions for FSRQs and BL Lacs (model LDDE 1 in Ajello et al. 2014) can reproduce the 1pPDF measurement fairly well in all five energy bands, as demonstrated in Figure 1. Misaligned AGNs enter the overall $d N / d S$ distribution below the threshold of the current analysis and therefore cannot be constrained (see Figure 1). Due to their intrinsic faintness, SFGs start to contribute only at very low, unconstrained fluxes.

However, Figure 2 shows that deviations in the faint end predicted by the other blazar models may be in tension with the allowed region derived from the $1 \mathrm{pPDF}$ analysis. A comprehensive study of the implications for blazar models is beyond the scope of this Letter, but we can conclude that the methodology of using the 1pPDF for measuring the gammaray source-count distribution has excellent sensitivity for probing unresolved blazars and the faint part of the blazar GLF.

We acknowledge the valuable support by the internal referee Marco Ajello and the anonymous journal referee in improving

\footnotetext{
9 The values assume reference model A of Ackermann et al. (2015b).
}

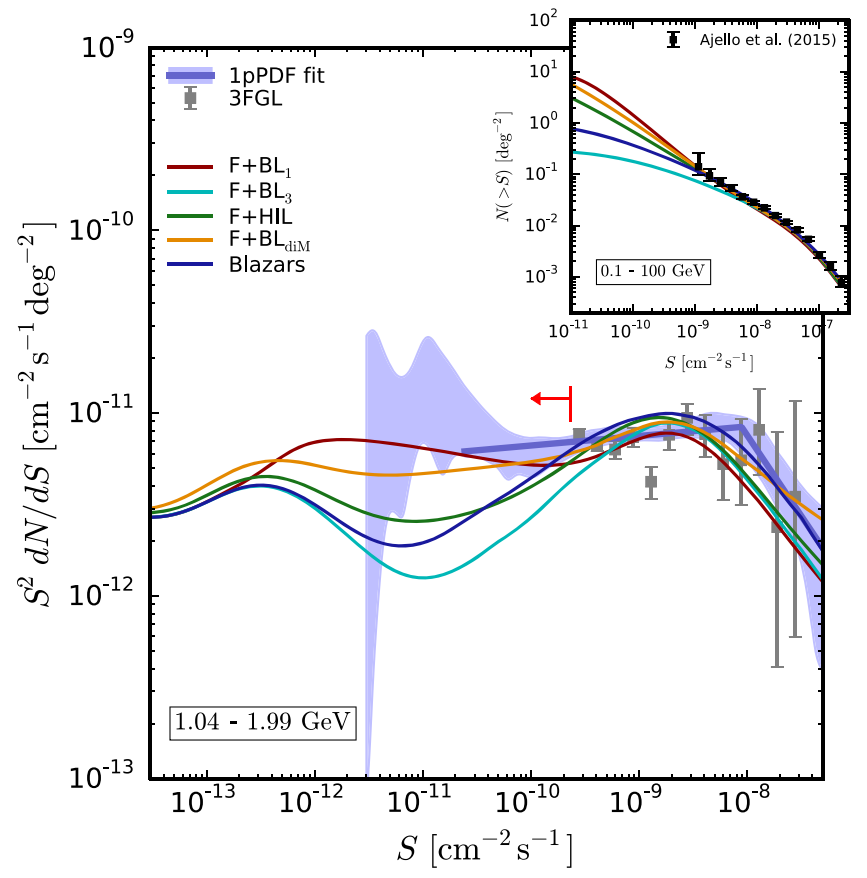

Figure 2. Differential source-count distribution $d N / d S$ in the $1.04-1.99 \mathrm{GeV}$ band, compared to blazar models. The three models $\mathrm{F}+\mathrm{BL}_{1}$ (solid red line), $\mathrm{F}$ $+\mathrm{BL}_{3}$ (solid cyan line), and $\mathrm{F}+\mathrm{HIL}$ (solid green line) include the sum of FSRQs (Ajello et al. 2012) and BL Lacs, which have been modeled assuming the luminosity functions $\mathrm{LDDE}_{1}, \mathrm{LDDE}_{3}$, and $\mathrm{LDDE}_{\mathrm{HSP}}+\mathrm{LDDE}_{\mathrm{ISP}+\mathrm{LSP}}$ of Ajello et al. (2014), respectively. The solid orange line shows the sum of the FSRQ component from Ajello et al. (2012) and the BL Lac model from Di Mauro et al. (2014c). The model depicted by the solid dark blue line refers to a single description of all blazars by Ajello et al. (2015). A contribution from mAGNs (Di Mauro et al. 2014a) has been added to each model. The vertical red line indicates the upper limit $(95 \% \mathrm{CL})$ on a second intrinsic break. The inset compares the integral source-count distribution $N(>S)$ between 0.1 and $100 \mathrm{GeV}$ (see Ajello et al. 2015) to the models (not including mAGNs).

the manuscript, and the support of the Servizio Calcolo e Reti of the Istituto Nazionale di Fisica Nucleare, Sezione di Torino.

This work is supported by the research grant Theoretical Astroparticle Physics number 2012CPPYP7 under the program PRIN 2012 funded by the Ministero dell'Istruzione, Università e della Ricerca (MIUR), by the research grants TAsP (Theoretical Astroparticle Physics) and Fermi funded by the Istituto Nazionale di Fisica Nucleare (INFN), and by the Strategic Research Grant: Origin and Detection of Galactic and Extragalactic Cosmic Rays as well as Excellent Young PI Grant: The Particle Dark-matter Quest in the Extragalactic Sky funded by Torino University and Compagnia di San Paolo.

The Fermi-LAT Collaboration acknowledges support for LAT development, operation and data analysis from NASA and DOE (United States), CEA/Irfu and IN2P3/CNRS (France), ASI and INFN (Italy), MEXT, KEK, and JAXA (Japan), and the K.A. Wallenberg Foundation, the Swedish Research Council and the National Space Board (Sweden). Science analysis support in the operations phase from INAF (Italy) and CNES (France) is also gratefully acknowledged.

\section{REFERENCES}

Abdo, A. A., Ackermann, M., Ajello, M., et al. 2010, ApJ, 720, 435 Acero, F., Ackermann, M., Ajello, M., et al. 2015, ApJS, 218, 23 Acero, F., Ackermann, M., Ajello, M., et al. 2016, ApJS, 223, 26 Ackermann, M., Ajello, M., Albert, A., et al. 2012a, ApJS, 203, 4 Ackermann, M., Ajello, M., Albert, A., et al. 2016a, PhRvL, 116, 151105 
Ackermann, M., Ajello, M., Albert, A., et al. 2015, ApJ, 799, 86 Ackermann, M., Ajello, M., Allafort, A., et al. 2012b, ApJ, 755, 164 Ackermann, M., Ajello, M., Allafort, A., et al. 2013, ApJS, 209, 34 Ackermann, M., Ajello, M., Atwood, W. B., et al. 2016b, ApJS, 222, 5 Ackermann, M., et al. 2012c, PhRvD, 85, 083007

Ajello, M., Gasparrini, D., Sánchez-Conde, M., et al. 2015, ApJL, 800, L27

Ajello, M., Romani, R. W., Gasparrini, D., et al. 2014, ApJ, 780, 73

Ajello, M., Shaw, M. S., Romani, R. W., et al. 2012, ApJ, 751, 108

Atwood, W. B., Abdo, A. A., Ackermann, M., et al. 2009, ApJ, 697, 1071

Cuoco, A., Komatsu, E., \& Siegal-Gaskins, J. M. 2012, PhRvD, 86, 063004

Di Mauro, M., Calore, F., Donato, F., Ajello, M., \& Latronico, L. 2014a, ApJ, 780, 161

Di Mauro, M., Cuoco, A., Donato, F., \& Siegal-Gaskins, J. M. 2014b, JCAP, 1411,021
Di Mauro, M., Donato, F., Lamanna, G., Sanchez, D. A., \& Serpico, P. D. 2014c, ApJ, 786, 129

Feroz, F., Hobson, M. P., \& Bridges, M. 2009, MNRAS, 398, 1601

Finke, J. D., Razzaque, S., \& Dermer, C. D. 2010, ApJ, 712, 238

Gehrels, N. 1986, ApJ, 303, 336

Górski, K. M., Hivon, E., Banday, A. J., et al. 2005, ApJ, 622, 759

Gruppioni, C., Pozzi, F., Rodighiero, G., et al. 2013, MNRAS, 432, 23

Lee, S. K., Lisanti, M., Safdi, B. R., Slatyer, T. R., \& Xue, W. 2016, PhRvL, 116,051103

Malyshev, D., \& Hogg, D. W. 2011, ApJ, 738, 181

Massaro, F., Thompson, D. J., \& Ferrara, E. C. 2016, A\&ARv, 24, 2

Ripken, J., Cuoco, A., Zechlin, H.-S., Conrad, J., \& Horns, D. 2014, JCAP, 1,049

Zechlin, H.-S., Cuoco, A., Donato, F., Fornengo, N., \& Vittino, A. 2016, ApJS, in press (arXiv:1512.07190) 